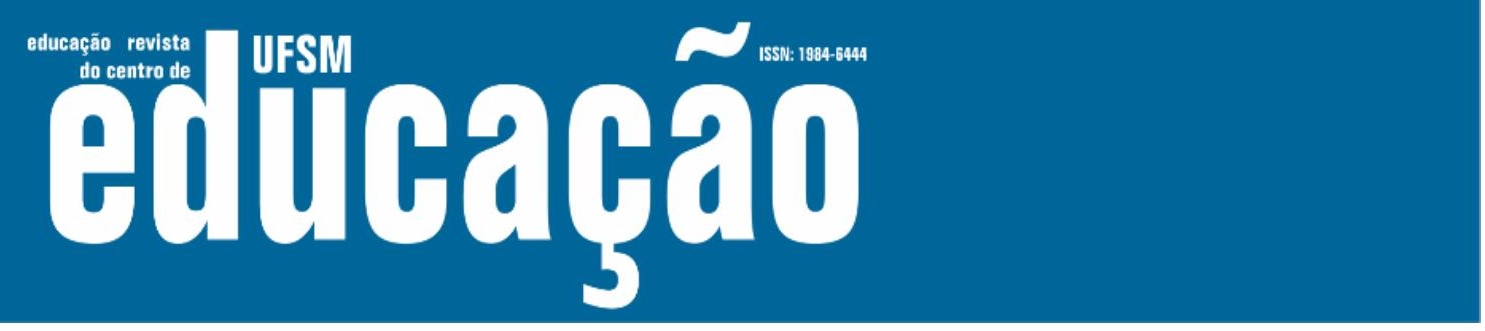

ISSN: 1984-6444 | http://dx.doi.org/10.5902/1984644442749

\title{
Potencialidades da iniciação científica no ensino superior para a formação docente
}

Potentialities of scientific initiation in higher education for teacher training

Potencialidades de la iniciación científica en la educación superior para la formación docente

Mayara Lopes de Freitas Lima

Mestra pela Universidade Federal Rural de Pernambuco, Recife, Pernambuco - Brasil maybiologicas@gmail.com - https://orcid.org/0000-0003-0231-3513

Eliane Matheus Plaza

Universidade Cruzeiro do Sul - São Paulo - Brasil

liaplaza@hotmail.com - https://orcid.org/0000-0001-5428-123X

Recebido em 06 de março de 2020

Aprovado em 01 de fevereiro de 2021

Publicado em 31 de julho de 2021

\section{RESUMO}

Considerando a relevância que têm adquirido os estudos acerca da Iniciação Científica no Ensino Superior, conforme pode ser visto na literatura especializada sobre o tema, pretende-se, neste artigo, analisar sua importância para a formação docente. Em função dessa análise, também se tem a intenção de apontar as potencialidades que podem ser desenvolvidas pelos futuros docentes e comparar as situações vivenciadas pelos pesquisados. $\mathrm{Na}$ investigação realizada, foram entrevistados 20 professores da carreira do Magistério público federal, todos com a titulação mínima de Mestrado, e o método utilizado foi o de análise do conteúdo. Os resultados indicaram que, dentre outras, as seguintes potencialidades: promover 0 domínio de métodos e técnicas de pesquisa, compreender todas as etapas de construção do conhecimento científico, preparar o futuro docente para que seja capaz de desenvolver pesquisas em nível de pós-graduação, dar segurança ao estudante de Iniciação Científica (IC) para que, ingressar no mundo do trabalho, ele possa enfrentar os desafios da sala de aula com inteligência emocional, dominando o conteúdo e compreendendo como o saber foi construído.

Palavras-chave: Iniciação Científica; Formação docente; Desenvolvimento científico. 


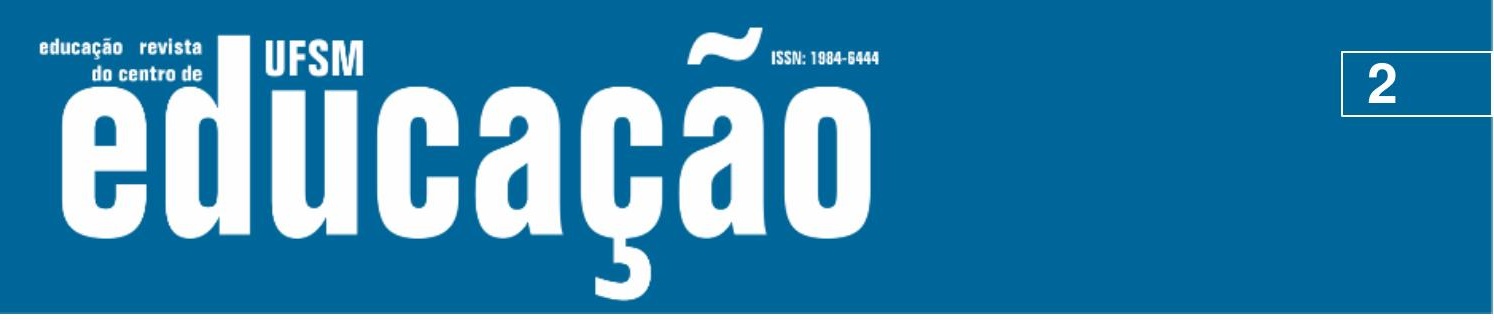

ISSN: 1984-6444 | http://dx.doi.org/10.5902/1984644442749

\section{ABSTRACT}

Considering the relevance that the studies about Scientific Initiation in Higher Education have acquired, as can be seen in the scientific literature, this article intends to analyze the importance of Scientific Initiation in Higher Education for teacher training. Due to this analysis, it is also intended to point out the potential that can be developed by future teachers, comparing the situations experienced by those surveyed. In the survey, 20 professors from the federal public teaching career were interviewed, all with a minimum master's degree and the method used was content analysis. According to our results, the following potentialities were evidenced, among others: promoting the mastery of research methods and techniques, understanding all stages of the construction of scientific knowledge, preparing the future teacher to be able to develop research at the postgraduate level, provide security to the Scientific Initiation $(\mathrm{Cl})$ student so that when entering the world of work he can face the challenges of the classroom with emotional intelligence, mastering the content and understanding how that knowledge was constructed.

Keywords: Scientific research initiation; Teacher training; Scientific development.

\section{RESUMEN}

Teniendo en cuenta la relevancia que han adquirido los estudios sobre la iniciación científica en la educación superior, como se puede ver en la literatura sobre el tema, este artículo pretende analizar la importancia de la iniciación científica en la educación superior para la formación del profesorado. Debido a este análisis, también se pretende señalar el potencial que pueden desarrollar los futuros docentes, comparando las situaciones experimentadas por los encuestados. En la encuesta, se entrevistó a 20 profesores de la carrera docente pública federal, todos con una maestría mínima y el método utilizado fue el análisis de contenido. Según nuestros resultados, se evidenciaron las siguientes potencialidades, entre otras: promover el dominio de los métodos y técnicas de investigación, comprender todas las etapas de la construcción del conocimiento científico, preparar al futuro maestro para poder desarrollar la investigación a nivel de posgrado, brinde seguridad al estudiante de iniciación científica (IC) para que al ingresar al mundo laboral pueda enfrentar los desafíos del aula con inteligencia emocional, dominar el contenido y comprender cómo se construyó ese conocimiento.

Palabras-clave: Iniciación Científica; Formación del profesorado; Desarrollo científico. 


\section{N-Tism

ISSN: 1984-6444 | http://dx.doi.org/10.5902/1984644442749

do tempo médio dessas titulações gerou transtornos para os respectivos programas de pós-graduação.

Nesse período, ainda havia uma peculiaridade sobre a concessão de bolsas de IC, que eram distribuídas via demanda espontânea ou balcão, porém com a solicitação do pesquisador (BIANCHETTI et al., 2012, p. 571; MASSI; QUEIROZ, 2010, p. 175). Isso significa que os pedidos de bolsas eram julgados por Comitês Assessores e concedidos por intermédio de cotas aos pesquisadores, os quais escolhiam os bolsistas.

Esse panorama mudou quando, em 1988, o CNPq criou o Programa Institucional de Bolsas de Iniciação Científica (Pibic). No âmbito desse programa, as bolsas passaram a ser concedidas diretamente às Instituições de Ensino Superior (IES) e aos Institutos de Pesquisa (IPq), que se tornaram responsáveis diretamente pelo controle administrativo dessas cotas e pela criação de dispositivos próprios para distribuí-las (MASSI; QUEIROZ, 2010, p. 175-176).

Com a criação do Pibic, em 1988, a Iniciação Científica foi normatizada e passou a ser considerada um programa voltado para o desenvolvimento do pensamento científico e iniciação à pesquisa de estudantes de graduação do ensino superior. Sobre isso, os autores afirmam:

IC é um meio de formação por excelência, pois permite introduzir os estudantes de graduação à pesquisa, colocando-os em contato com a atividade científica. Pode ser também uma estratégia de base teóricometodológica que auxilia no processo de realização da pesquisa, além de constituir-se em uma via adequada de contribuição à construção de uma nova mentalidade do estudante no que diz respeito à relação ensino e pesquisa; graduação e pós-graduação (e, atualmente, Educação Básica) (BIANCHETTI et al., 2012, p. 572).

Os dados fornecidos pelo CNPq e publicados na literatura científica mostram que, com o advento do Pibic, houve um crescimento substancial no número de bolsas ofertadas. Apesar de ter sido um período de oscilação entre 1995 e 2003, os números são bastante satisfatórios, como pode ser visto nos gráficos apresentados, por exemplo, nos trabalhos de Nogueira e Canaan (2009, p. 44), Bianchetti et al. (2012, p. 575) e Massi e Queiroz (2014). 


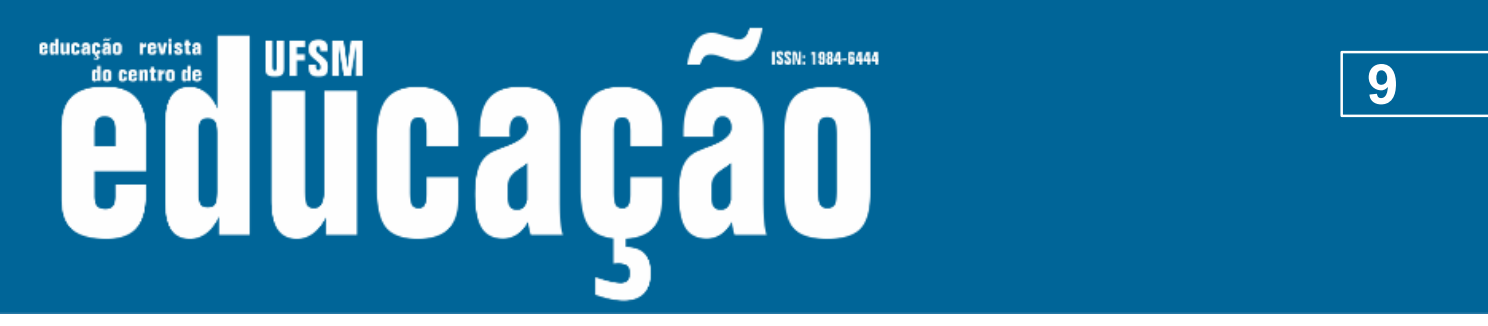

ISSN: 1984-6444 | http://dx.doi.org/10.5902/1984644442749

do Pibic. A partir daí, houve um crescimento até 1995, seguido por uma oscilação que durou até 2003. Nesse período, o país foi guiado por uma política neoliberal e, apesar da estabilidade da moeda, a IC não parece ter sido valorizada. No período seguinte (2003-2014), houve um crescimento contínuo no número de bolsas, tanto que a quantidade de bolsas ofertadas em 2014 (26.970) foi de quase 50\% a mais do que em 2003 (18.238). Obviamente, é necessário aumentar esses números, mas o fato de haver crescimento já indica uma política de preocupação com a qualidade da formação do graduando. A figura 1 mostra a evolução do número de bolsas de IC ao longo dessa série temporal.

Figura 1 - Número de bolsas de Iniciação Científica (Série histórica 1976 - 2014)

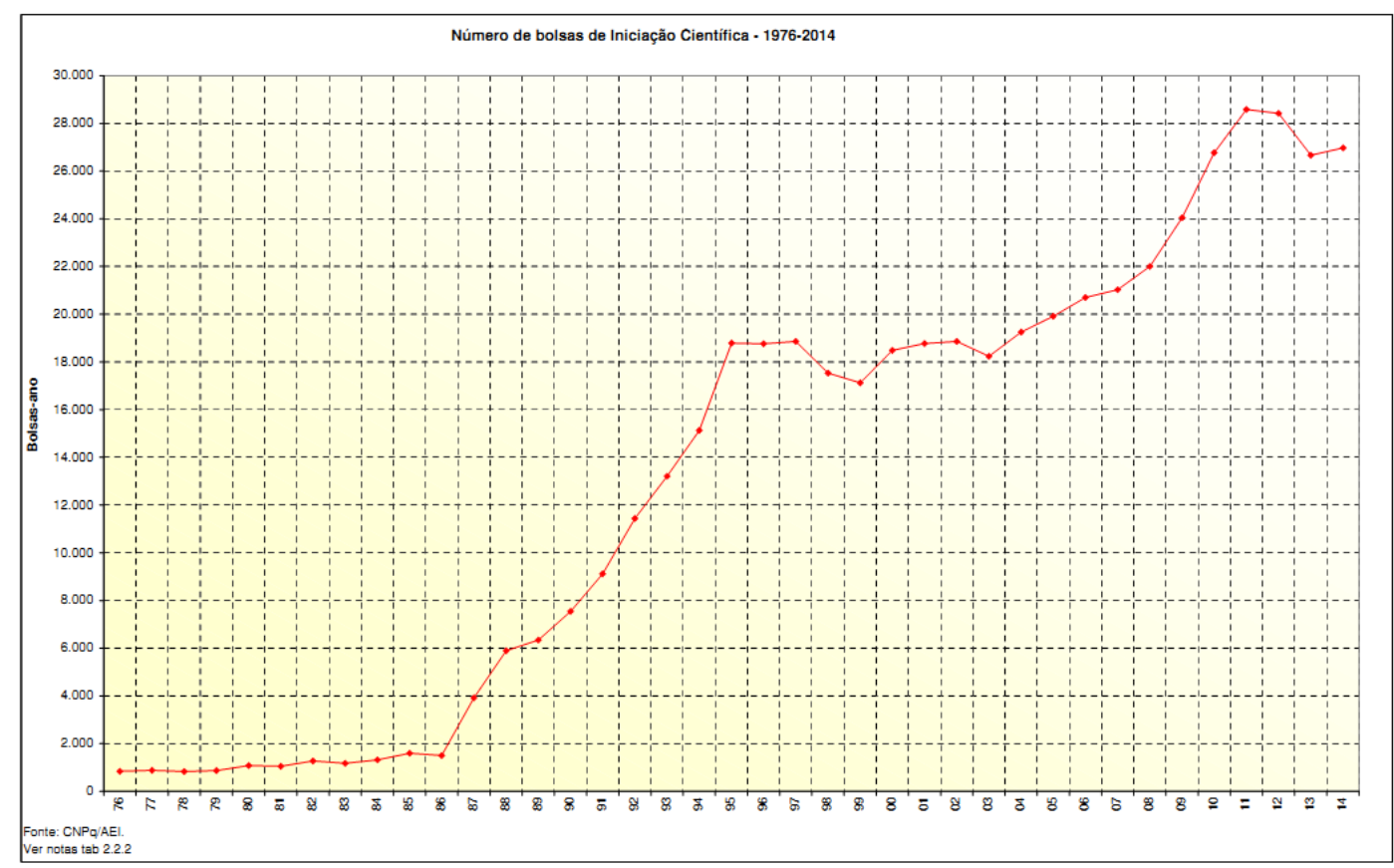

Fonte: CNPq. (2019b)

Nesta investigação, em que se foi realizada a optou por estudos com abordagem qualitativa, foram feitos levantamento bibliográfico e entrevistas com profissionais que tiveram experiências práticas com o referido problema pesquisado, o que possibilita analisar de exemplos que estimulam a compreensão da discussão (PRODANOV; FREITAS, 2013, p. 52). Nesse tipo de pesquisa, a interpretação dos 


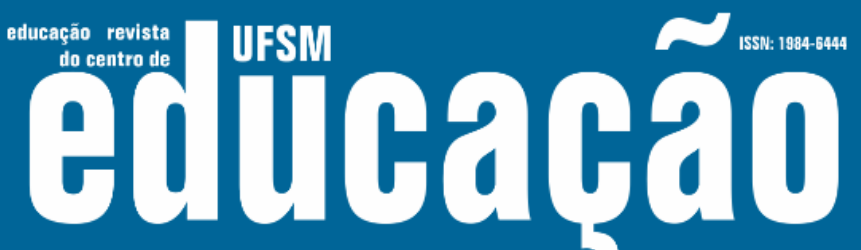

ISSN: 1984-6444 | http://dx.doi.org/10.5902/1984644442749

fenômenos e a atribuição de significados são condições básicas (SILVA; MENEZES, 2001, p. 19).

Oliveira (2011, p. 43) afirma que um instrumento de pesquisa é considerado válido quando consegue medir, com precisão, o que se deseja conhecer. Como, neste estudo, pretendeu-se analisar a importância da Iniciação Científica no Ensino Superior para a formação docente, exigiu-se um instrumento confiável e preciso. Para isso, foram utilizados dois questionários, cujo conteúdo se encontra no apêndice deste artigo. Entre as perguntas realizadas, destacam-se questionamentos sobre a opinião dos pesquisados acerca do impacto de sua participação ou não em programas de Iniciação Científica em suas Graduações e no Mestrado.

$\mathrm{Na}$ pesquisa realizada, foram entrevistados 20 professores do Magistério público federal, todos com a titulação mínima de Mestrado. No universo pesquisado, espera-se atingir professores com várias formações e tempo de experiência profissional, proporcionando uma riqueza na coleta de dados para assegurar a confiabilidade dos resultados.

Para analisar os dados coletados, foi empregada a técnica de análise do conteúdo (BARDIN, 2011), um método muito utilizado em pesquisas qualitativas, por meio do qual se busca, a partir das respostas dos entrevistados às perguntas do questionário, produzir inferências, ou seja, fazer operações lógicas, em que se admite uma proposição em virtude de sua ligação com outras já aceitas como verdadeiras (CAMPOS, 2004, p. 613).

\section{Resultados e discussão}

Os questionários foram respondidos por 20 docentes do Magistério público federal, todos com a titulação mínima de Mestrado. Dentre eles, 55\% participaram de programas de IC, e 45\% não tiveram essa oportunidade. Com relação ao sexo dos entrevistados, $55 \%$ são do sexo masculino, e $45 \%$, do feminino. Quanto à faixa etária, a figura 2 resume as informações: 10\% (até 30 anos), 55\% (entre 31 e 50 anos) e $35 \%$ (acima de 50 anos). 


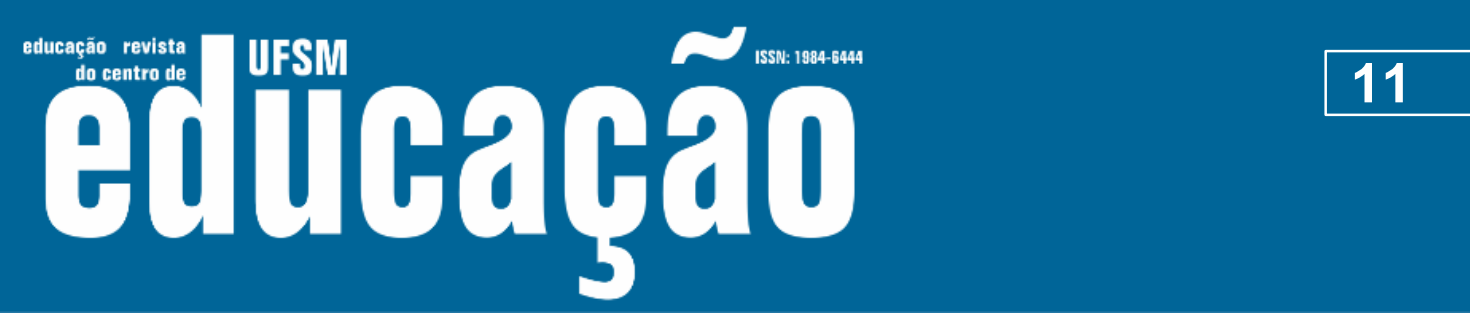

ISSN: 1984-6444 | http://dx.doi.org/10.5902/1984644442749

Figura 2- Faixa etária dos docentes entrevistados

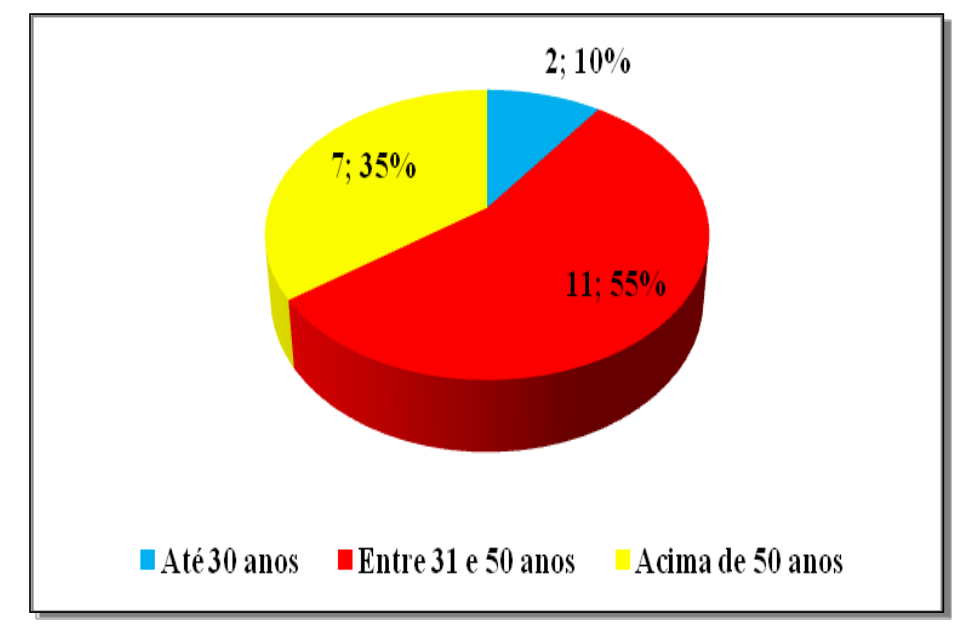

Fonte: Elaborado pelas autoras

Aos docentes que fizeram IC foi perguntado como avaliam sua importância em sua formação na graduação. Todos declararam que foi consideravelmente importante. Seguem as respostas de três deles:

Quadro 1 - Respostas dos entrevistados sobre a importância da IC na Graduação

Fundamental! A partir da IC, obtive encaminhamentos para continuar meus estudos acadêmicos. Foi durante a IC que decidi a linha de pesquisa do mestrado e, vale dizer, não foi a mesma dos estudos que desenvolvi na IC.

Muito importante. Comecei a IC na Universidade no segundo ano de curso. Isso me proporcionou participar e integrar um grupo de pesquisa com vários professores, estudantes de Pós-Graduação, professores e alunos visitantes, que, além de permitir a comunicação em língua estrangeira efetivamente me deu a certeza do trabalho desenvolvido por um cientista da minha área.

A Iniciação Científica nos permite ter acesso à pesquisas em diferentes dimensões, desde à familiarização com os processos de pesquisa à abertura de oportunidades no meio acadêmico.

Fonte: Elaborado pelas autoras - 2020

Aos docentes que não fizeram IC na graduação, foi perguntado se sentiram falta, ao longo de sua formação na graduação, de não ter participado de Programas de IC. Em suas respostas, 77,8\% deles admitiram ter sentido falta, e apenas $22,2 \%$ responderam não ter sentido falta. 


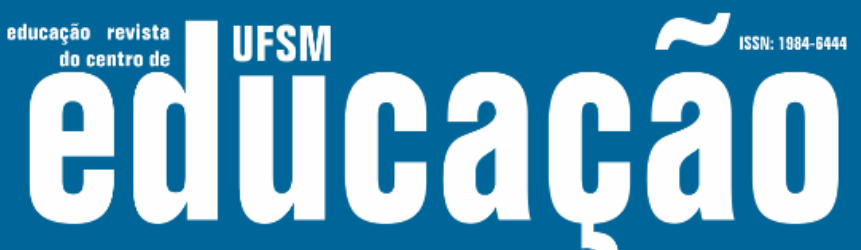

ISSN: 1984-6444 | http://dx.doi.org/10.5902/1984644442749

Quanto aos docentes que participaram de programas de IC, $72,7 \%$ admitem que sua participação nesses programas facilitou sua entrada no Mestrado, e 100\% dos que não participaram de programas de IC acreditam que, se tivessem feito IC, sua aprovação no Mestrado teria sido facilitada. Esse resultado corrobora o pensamento de Nogueira e Canaan (2009, p. 50) de que há uma correlação direta entre o estudante ter sido bolsista de Iniciação Científica nos mais diferentes cursos de graduação e Instituições de Ensino Superior e sua posterior entrada nos cursos de pós-graduação stricto sensu.

Estudos realizados demonstram que o número de alunos egressos dos Programas de Iniciação Científica que ingressam nos cursos de pós-graduação stricto sensu é proporcionalmente bem maior do que o dos que não passaram por essa experiência. Pires (2007, p. 130) esclarece que uma avaliação feita pelo CNPq acerca do investimento realizado no Pibic, na década de 1990, indicou que houve um aproveitamento satisfatório, porque o resultado indicou que a probabilidade de um graduado, cuja bolsa PIBIC tenha terminado no ano de sua formatura, chegar ao Mestrado foi bem mais considerável $(37 \%)$ do que a dos não bolsistas $(5 \%)$ (ARAGÓN, 1999, p. 33, apud PIRES, ibid). Esse resultado também foi constatado por Nogueira e Canaan (2009, p. 66), que, em seus resultados, notaram que os bolsistas de IC tiveram mais acesso aos programas de pós-graduação stricto sensu do que os que não fizeram essas atividades.

Nesta pesquisa, apenas $66,7 \%$ dos docentes que não fizeram IC acreditam que o fato de não terem participado desses programas influenciou o tempo de conclusão do seu Curso de Mestrado. A seguir, apresentam-se três das respostas dadas pelos que pensam assim: 


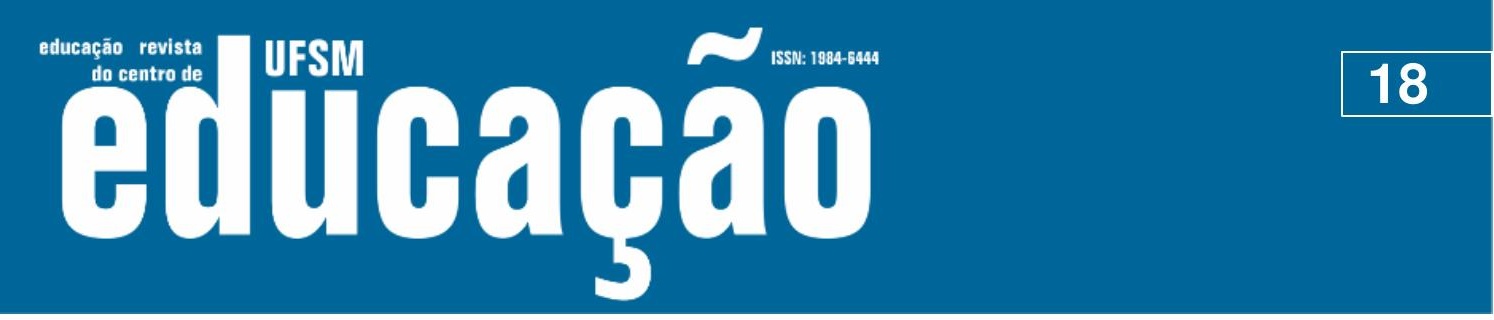

ISSN: 1984-6444 | http://dx.doi.org/10.5902/1984644442749

d) Aumentar os estímulos para uma formação criativa, crítica e autônoma;

e) Preparar o futuro docente para que seja capaz de desenvolver pesquisas em nível de pós-graduação;

f) Proporcionar segurança ao estudante de IC, para que, este ao ingressar no mundo do trabalho, ele possa enfrentar os desafios da sala de aula com inteligência emocional, dominando o conteúdo e compreendendo como o saber foi construído.

É importante ressaltar que este artigo abre perspectivas para que novas pesquisas sejam realizadas com outros públicos e, possivelmente, até graduandos dos cursos de licenciatura, para se compreender como eles veem as potencialidades da IC em sua formação. Como não foi possível comparar públicos variados, a investigação se limitou a uma análise mais abrangente. Todavia, devido às especificidades da pesquisa, pode-se deixar essa lacuna, que poderá ser preenchida por futuros trabalhos que objetivem compreender o tema com mais profundidade.

\section{Referências}

ARAGÓN, Virgílio Alvarez (org.). O Programa Institucional de Bolsas de Iniciação Científica (PIBIC) e sua relação com a formação de cientistas. Relatório Final. Brasília: UnB/NESUB, 1999.

BARDIN, Laurence. Análise de conteúdo. São Paulo: Edições 70, 2011.

BAZIN, Maurice Jacques. O que é a Iniciação Científica. Revista Brasileira de Ensino de Física, v. 5, n. 1, p. 81-88, jun. 1983. Disponível em: http://www.sbfisica.org.br/rbef/indice.php?vol=5\&num=1. Acesso em 25 ago. 2019.

BERBEL, Neusi Aparecida Navas. As metodologias ativas e a promoção da autonomia de estudantes. Semina: Ciências Sociais e Humanas, v. 32, n. 1, p. 25-40, jan./jun. 2011. Disponível em: http://dx.doi.org/10.5433/1679-0383.2011v32n1p25. Acesso em 24 jan. 2021.

BIANCHETTI, Lucídio et al. A iniciação à pesquisa no Brasil: políticas de formação de jovens pesquisadores. Educação, v. 37, n. 3, p. 569-584, set./dez. 2012. Disponível em: https://periodicos.ufsm.br/reveducacao/article/view/5012. Acesso em 22 ago. 2019. 


\section{uss \\ 1SSN: $1984-6444$

ISSN: 1984-6444 | http://dx.doi.org/10.5902/1984644442749

VILLAS BÔAS, Gláucia K. Currículo, iniciação científica e evasão de estudantes de Ciências Sociais. Tempo Social, v. 15, n. 1, p. 45-62, abr. 2003. Disponível em: http://www.scielo.br/scielo.php?script=sci_arttext\&pid=S0103-20702003000100003. Acesso 22 ago. 2019.

VON ZUBEN, Newton Aquiles. A relevância da iniciação à pesquisa científica na universidade. Pro-posições, v. 6, n. 2, p. 5-18, 1995. Disponível em: https://periodicos.sbu.unicamp.br/ojs/index.php/proposic/article/view/8644266.

Acesso em 22 ago. 2019. 


\section{$\sim$

ISSN: 1984-6444 | http://dx.doi.org/10.5902/1984644442749

\section{QUESTIONÁRIO DE COLETA 1 - PARTICIPANTES DE PROGRAMAS DE INICIAÇÃO CIENTÍFICA}

Caro(a) colega:

Inicialmente, gostaria de agradecer pela sua disponibilidade em cooperar com o presente trabalho acadêmico. Sua contribuição será anônima e o resultado deste questionário só será utilizado com fins acadêmicos.

Sexo: ( ) Feminino

( ) Masculino

Idade:

( ) Até 30 anos

( ) 31 a 50 anos

( ) 50 anos em diante

1. Qual a avaliação que você faz da importância da IC em sua formação (na graduação)?

2. Ter participado de programas de IC facilitou sua aprovação no Mestrado?
( ) SIM
( ) NÃO

3. Em quantos meses você concluiu o Mestrado?

4. Você acredita que ter participado de programas de IC ajudou a concluir seu Curso de Mestrado em menos tempo? Explique.

5. A participação em programas de IC influenciou o desenvolvimento de suas pesquisas no Mestrado? Justifique sua resposta.

6. Em sua opinião, fazer ou não fazer Iniciação Científica na graduação é relevante para a formação do docente?

7. Fique à vontade para apresentar suas impressões sobre os programas de IC. 


\section{-7= usm,

ISSN: 1984-6444 | http://dx.doi.org/10.5902/1984644442749

\section{QUESTIONÁRIO DE COLETA 2 - NÃO PARTICIPANTES DE PROGRAMAS DE INICIAÇÃO CIENTÍFICA}

Caro(a) colega:

Inicialmente, gostaria de agradecer pela sua disponibilidade em cooperar com o presente trabalho acadêmico. Sua contribuição será anônima e o resultado deste questionário só será utilizado com fins acadêmicos.

Sexo: ( ) Feminino

( ) Masculino

Idade:

( ) Até 30 anos

( ) 31 a 50 anos

( ) 50 anos em diante

1. Você sentiu, ao longo de sua formação na graduação, falta de não ter participado de programas de IC?

( ) SIM

( ) NÃO

2. Você acredita que, se tivesse participado de programas de IC, teria facilitado sua aprovação no Mestrado?

( ) SIM ( ) NÃO

3. Em quantos meses você concluiu o Mestrado?

4. Você acredita que não ter participado de programas de IC influenciou o tempo de conclusão do seu Curso de Mestrado? Explique.

5. A não participação em programas de IC influenciou no desenvolvimento de suas pesquisas no Mestrado? Justifique sua resposta.

6. Em sua opinião, fazer ou não fazer Iniciação Científica na graduação é relevante para a formação do docente?

7. Fique à vontade para apresentar suas impressões sobre os programas de IC. 


\section{Lew \\ ISSN: 1984-6444

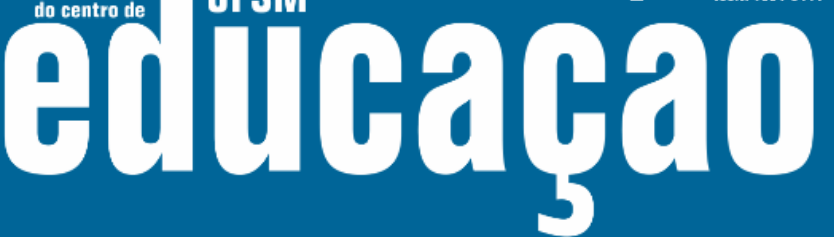

ISSN: 1984-6444 | http://dx.doi.org/10.5902/1984644442749

\section{(C) $(1) \otimes$}

This work is licensed under a Creative Commons Attribution-NonCommercial 4.0 International (CC BY-NC 4.0)

\section{Nota}

${ }^{1}$ A Resolução Normativa RN 017/2006 é o marco que estabelece as normas gerais e específicas para a concessão das diversas modalidades de bolsas por quota, no país, por parte do CNPq. 